Culture et histoire dans l'espace roman

\title{
Les empreintes freudiennes dans Sinrazón d'Ignacio Sánchez Mejías (1928)
}

David Coste

\section{(2) OpenEdition}

1 Journals

Édition électronique

URL : https://journals.openedition.org/cher/11588

DOI : $10.4000 /$ cher. 11588

ISSN : 2803-5992

Éditeur

Presses universitaires de Strasbourg

\section{Édition imprimée}

Date de publication : 1 décembre 2012

Pagination : 145-156

ISBN : 978-2-35410-049-0

ISSN : 1968-035X

\section{Référence électronique}

David Coste, «Les empreintes freudiennes dans Sinrazón d'Ignacio Sánchez Mejías (1928) »,

reCHERches [En ligne], 9 | 2012, mis en ligne le 14 février 2022, consulté le 16 février 2022. URL : http:// journals.openedition.org/cher/11588; DOI : https://doi.org/10.4000/cher.11588

\section{(c) (†) (2)}

Ce(tte) œuvre est mise à disposition selon les termes de la Licence Creative Commons Attribution Pas d'Utilisation Commerciale - Partage dans les Mêmes Conditions 4.0 International. 


\title{
Les empreintes freudiennes dans Sinrazón d'Ignacio Sánchez Mejías (1928)
}

\author{
DAVID COSTE \\ Université de Strasbourg
}

\begin{abstract}
T'introduction des théories psychanalytiques freudiennes dans le monde Lintellectuel espagnol des années $1920^{1}$ révolutionna la conception que l'on avait jusqu'alors de la psychologie humaine. En Espagne, comme ailleurs, le fou, dont l'anormalité était au Moyen Âge d'origine divine avant d'être le fruit d'un dérèglement des humeurs corporelles à la Renaissance, est alors appréhendé à travers le prisme de la médecine psychanalytique comme un malade psychotique. Ce nouveau statut de la folie dans la société a des répercussions considérables en littérature. En effet, le personnage du dément dans le théâtre espagnol de la fin des années 1920 ne sort pas indemne de ces bouleversements de la pensée.
\end{abstract}

Le premier galop d'essai littéraire d'Ignacio Sánchez Mejías, qui était jusque-là connu pour ses prouesses dans l'arène, préfigure une nouvelle veine théâtrale qui témoigne de cette empreinte freudienne dans un théâtre qui est alors en crise et cherche à explorer de nouveaux horizons.

Le premier acte de Sinrazón se situe dans l'asile que dirige le docteur Ballina. Ce dernier explique dès le début de la pièce comment, grâce à la théorie psychanalytique qu'il a appliquée sur son riche patient Don Manuel, il a pu le sauver de la démence. L'action se situe donc au moment où Don Manuel est sur le point de quitter l'asile pour réintégrer la société. La fin $\mathrm{du}$ premier acte scelle une promesse entre Ballina et Don Manuel qui souhaite employer sa fortune dans la construction d'un nouvel asile où le

1 L’intégralité des théories freudiennes sont publiées en 1992 en Espagne. 
terme de fou serait à bannir. Le deuxième acte donne vie au projet de Don Manuel et le "Palacio de la Reina Beatriz» permet à ses hôtes de vivre dans l'illusion en leur donnant les moyens d'être ce qu'ils se figurent être. La reine Beatriz apparaît avec sa cour et vêtue d'un manteau d'hermine et d'une véritable couronne royale. Tous les fous s'enfoncent alors dans leur délire psychotique qui devient plus vrai que nature. Le docteur Ballina prend peu à peu conscience de son erreur et de la distance grandissante qui sépare les malades d'une hypothétique guérison. Le troisième acte, qui a lieu dans le même espace, consacre les divergences d'opinion qui se creusent entre Ballina et Don Manuel et qui préfigurent la catastrophe finale. La reine accepte de ne plus se croire reine. Au moment où cette dernière est sur le point d'avouer au docteur l'attirance qu'elle a envers lui, Don Manuel, jaloux et contrarié par ce revirement soudain, étrangle le docteur Ballina qui meurt sur scène. Le rideau tombe sur un asile qui sombre de plus belle dans la folie.

Si la seule évocation de l'intrigue de Sinrazón ne laisse aucun doute quant à l'ampleur de l'empreinte freudienne dans ce théâtre, comment et à quels niveaux s'exprime-t-elle dans l'œuvre? Est-on face à un théâtre à thèse, de simple vulgarisation d'une pensée théorique ou, au contraire, cette marque freudienne est-elle le signe d'un scepticisme du milieu intellectuel espagnol de l'époque face à cette nouvelle inconnue?

\section{L'empreinte dramatique}

Le personnage de Ballina, porte-parole de la psychanalyse dans l'œuvre

Le déterminisme psychanalytique au $\mathrm{xx}^{\mathrm{e}}$ siècle enracine la démence dans un vécu traumatique qui se construit dès l'enfance. Le «fou» est alors celui qui ne parvient pas à résoudre le conflit psychique entre les différentes aires de sa psychologie; autrement dit, entre son Moi ou partie consciente, son Ça ou part inconsciente et son Surmoi ou censure morale. Ce bagage théorique freudien apparaît clairement dans la pièce à travers le personnage de Ballina qui est un véritable porte-parole de la psychanalyse freudienne: «[...] le hablé del inconsciente, de la censura que forma la moral, de las luchas entre la conciencia y la inconsciencia, de que su propia enfermedad demostraba su hombría...» (Sánchez Mejías 1988: 60). La terminologie freudienne affleure de toutes parts, notamment dans l'explication sexuelle du traumatisme psychotique: "quedé plenamente convencido que estaba bajo la influencia de un choque producido por un sentimiento perverso de la sexualidad». (Sánchez Mejías 1988: 60) 
L'espace dramatique semble alors, dans une première lecture, devenir l'espace de vulgarisation de la nouvelle conception de la psychologie humaine. Les grands traits de la théorie freudienne du rêve, détaillée dans Le rêve et son interprétation (Freud 1985), apparaissent synthétisés en quelques mots dans la bouche du docteur:

El sueño, según teorías modernas, es un deseo reprimido por nuestra consciencia. En la realización de este deseo se toman incoherentemente materiales en nuestra vida, relacionados con el mismo deseo, pero, al despertar, el olvido borra lo que soñamos, o es rechazado nuestro sueño por las reglas de la normalidad. Ahora bien; nuestra infancia y nuestra juventud están llenas de deseos, unos lógicos y naturales, otros morbosos y perversos, la mayoría de ellos inconfesables. Nuestra moral, haciendo de censura, se encarga de rechazarlos, y en esta lucha entre el deseo y la censura está la llave de la mayoría de las perturbaciones. (Sánchez Mejías 1988: 72)

\section{La transposition dramatique de concepts psychanalytiques}

Au niveau dramatique, l'empreinte freudienne est bien plus prégnante et va bien au-delà de la citation implicite. La structure même de l'œuvre met en avant les découvertes freudiennes sur le psychisme humain.

\section{Conflit psychique et écriture de la cure psychanalytique}

Le conflit psychique des personnages est au cœur de l'écriture dramatique comme en témoigne, au début du troisième acte, la cure psychanalytique dont est l'objet le fou qui se prend pour un évêque. La scène donne à voir et à entendre les concepts freudiens «d'associations libres » et de "résistances» dans cette transposition dramatique de la psychothérapie. Le premier apparaît dans l'insistance du docteur pour que le patient exprime tout ce qui lui vient à l'esprit:

Sigue, sigue $[\ldots]$ ¿Tu recuerdas las cosas de tu niñez? [...] Contéstame a todo lo que te pregunte. Sin recelos [...] Vamos a ver. Cierra los ojos. Concentra tu atención en Manolo Isla, y vas diciendo espontáneamente todo lo que vayas pensando al recordar ese nombre (Sánchez Mejías 1988: 82-84)

Les réponses de l'évêque soulignent les résistances du patient sous l'effet du refoulement psychique: "Ballina. Sigue/ Obispo (Con intención) No. [...] Nada, no me acuerdo de nada. [...] No. ¿Para qué? Es que... no merece la pena.» (Sánchez Mejías 1988: 82-83). 
On assiste donc à l'écriture de la cure psychanalytique comme l'entend Freud et sans que ce dernier ne soit explicitement cité2. L'écriture dramatique traduit alors la force de l'inconscient et des désirs inconfessables, aux prises avec le refoulement provoqué par la morale. Les silences que suggèrent les points de suspension sont autant de marques de ce conflit intérieur à l'origine du trouble pathologique.

\section{Le Complexe œdipien}

Autre grand item de la psychanalyse freudienne, le complexe œedipien sert d'explication dans l'œuvre au délire de celle qui se croit reine, comme l'avait déjà fait remarquer Carlos Blanco Dávila (Blanco Dávila 2000). De manière schématique, il s'agit du désir sexuel pour le parent de sexe opposé qui génère la haine du parent de même sexe. La clef freudienne qui explique le comportement de la patiente apparait de nouveau en filigrane: "Il est des symboles à interprétation unique; ainsi Empereur et Impératrice, Roi et Reine, signifient Père et Mère...» (Freud 1985: 114). Or depuis le début de la pièce, le personnage rappelle sans cesse qu'elle attend son roi qui ne vient jamais. Les paroles de la reine du deuxième acte confirment l'idée du complexe œdipien: "El médico me ha dicho que yo no soy reina; que quiero serlo porque me enamoré siendo una niña del Rey, y quiero casarme con él.» (Sánchez Mejías 1988: 78)

\section{De la folie à la maladie psychotique: nouvelle représentation du fou}

L'empreinte freudienne dans Sinrazón se traduit par le passage du concept général de folie, à des maladies précises qui confèrent un caractère individuel à la folie.

Le délire paranoïaque fait l'objet d'une véritable transposition dramatique dans l'œuvre. La surestimation du moi, la méfiance ou le délire de persécution sont les symptômes récurrents du psychotique paranoïaque que l'on peut déceler chez les personnages de la reine ou l'évêque dans la pièce de Sánchez Mejías. Les répliques de ces derniers sont marquées par une

2 Ainsi, Freud affirme dans Cinq leçons sur la psychanalyse, (2001: 43): «Il arrive parfois qu'elle semble échouer: le malade s'arrête brusquement, hésite et prétend n'avoir rien à dire, qu'il ne lui vient absolument rien à l'esprit. S'il en était réellement ainsi, notre procédé serait inapplicable. Mais une observation minutieuse montre qu'un tel arrêt des associations libres ne se présente jamais. Elles paraissent suspendues parce que le malade retient ou supprime l'idée qu'il vient d'avoir, sous l'influence de résistance revêtant la forme de jugements critiques. On évite cette difficulté en avertissant le malade à l'avance et en exigeant qu'il ne tienne aucun compte de cette critique.» 
multiplication des pronoms personnels sujets, ce qui souligne, de manière non anodine, un égocentrisme pathologique: "Yo no me caso con nadie. Yo no quiero a nadie.» (Sánchez Mejías 1988: 67). Ou bien: «¿Yo qué soy? Si no soy obispo, ¿qué soy yo? Porque algo seré yo...» (Sánchez Mejías 1988: 81). La fin du premier acte donne à voir cette méfiance du paranoïaque à travers les propos de la reine: "que me están tendiendo lazos continuamente, que me tienen encerrada» (Sánchez Mejías 1988: 68). Ces craintes se transforment vite en un véritable délire de persécution: "Y hay gente interesada en que yo no me case. No sé por qué; pero la hay, la hay [...] La hay, la hay». (Sánchez Mejías 1988: 78)

La théorie du complot, marque d'une psychose paranoïaque, vise un ennemi toujours impersonnel. En espagnol, cela se traduit par une troisième personne du pluriel qui est récurrente dans les propos des deux personnages: "Es necesario que venga el Rey. Que venga pronto. Díselo tú. Que me quieren casar con un médico" (Sánchez Mejías 1988: 78). L'évêque est victime dans l'œuvre d'une même folie paranoïaque: "Todavía no me entregan mis documentos. Maniobras políticas de Roma [...] Ahora mismo me juegan una mala partida. Yo no sé quién. No sé quién» (Sánchez Mejías 1988: 76). Le délire est alors l'occasion d'une mise en scène de la cure psychanalytique qui révèle le rôle des songes dans la résolution du conflit psychique.

\section{L'empreinte dramaturgique}

\section{Du psychodrame au jeu de rêves}

La trame de Sinrazón qui établit le psychodrame, autrement dit le recours au théâtre dans une perspective thérapeutique, comme élément fondateur de l'intrigue, constitue un véritable pont entre l'écriture dramatique de la psychanalyse et son empreinte, moins décelable au niveau dramaturgique. Le psychodrame est à l'origine un traitement thérapeutique de la psychose qui se caractérise par une tentative de mise en scène de l'illusion du malade utilisée par le psychiatre afin de le guérir de ses obsessions, traumatismes ou fantasmes. En d'autres termes, il s'agit d'un jeu dramatique de scènes réelles ou imaginaires pratiqué à des fins thérapeutiques. Or, on retrouve ce même schéma dans Sinrazón qui consiste à entrer dans l'illusion du patient pour le soigner «de l'intérieur».

La structure même du psychodrame dans Sinrazón est un nouvel exemple de la transposition dramatique d'une pratique psychanalytique, comme en témoigne le passage du premier au deuxième acte. En effet, le 
psychodrame de Moreno obéit à trois étapes dont les deux premières se reflètent parfaitement dans l'œuvre de Sánchez Mejías. Il s'agit "d'abord de la mise en train ou l'échauffement du groupe, où le thérapeute, directeur $d u$ jeu a provoqué et excité les intervenants." (Thuillier 1996: 287). Dès la fin du premier acte, le docteur Ballina reprenant les paroles de son patient Don Manuel est l'instigateur du psychodrame qui prend vie au deuxième acte: "Vamos a crear una leyenda y a darle vida. Una reina, prisionera de amor, en su palacio espera al Rey para casarse». (Sánchez Mejías 1988: 63)

Mais au deuxième acte, l'écriture dramatique du psychodrame laisse place à une dramaturgie du jeu de rêve toute freudienne. En effet, le déguisement n'est plus seulement la marque de théâtre enchâssé ${ }^{3}$, ou psychodrame de Moreno; il outrepasse ce cadre et ouvre la voie à la «dramaturgie du jeu de rêve»; concept mis notamment en lumière par Jean-Pierre Sarrazac (Sarrazac, 2004: 57-68) et qui témoigne d'un élan vers l'onirique des dramaturgies du tournant du siècle en Europe.

Le deuxième acte, n'est autre que le rêve manifeste, c'est-à-dire le rêve tel qu'il apparaît dans la mémoire du dormeur Don Manuel, où s'expriment ses désirs cachés de pouvoir et de séduction.

Les coïncidences entre rêve et théâtre font de ce dernier le lieu privilégié de la projection des désirs refoulés du psychotique comme le souligne Freud:

Mais le rêve donne quelque chose de plus que ce mode optatif; il nous montre le souhait réalisé, il nous offre cette réalisation sous une forme réelle et actuelle; et les matériaux dont il se sert pour nous la représenter consistent le plus souvent en situations, en images sensorielles, presque toujours visuelles [...] il se produit une sorte de transposition que nous pouvons appeler travail de rêve; une pensée qui existait sous la forme optative est remplacée par une image actuelle. (Freud 1985: 38)

Le caractère condensé du rêve est l'expression visuelle, symbolique de nos pensées enfouies dans la théorie freudienne. Le rêve s'adapte donc parfaitement au théâtre en tant qu'art par excellence de l'impact visuel.

Dans cette dramaturgie du jeu de rêve, le déguisement folklorique est l'expression dramaturgique du déplacement de la réalité qui masque ou déguise le vécu sous l'effet du Surmoi. Tout le travail du rêve n'est autre qu'un

3 Jean Thuillier (1996: 287) explique que la deuxième étape du psychodrame de Moreno «va révéler l'action dramatique où le sujet principal va jouer, devant le groupe de patientspectateurs, la situation qui a été choisie; l'acteur principal est assisté de partenaires, qui peuvent en cours de séance changer de rôles.» 
processus de transformation, déguisement, d'idées réprimées par la censure morale que le costume de théâtre donne à voir de manière évidente.

Cette nouvelle dramaturgie est d'ailleurs annoncée dès le départ par cette voix nietzschéenne, didascalie irreprésentable, qui rappelle l'importance de la facette onirique de la réalité dans la conduite des hommes:

Aquello que vivimos en sueño, siempre que lo vivamos con frecuencia, pertenece al fin y al cabo, a la totalidad de nuestra alma, como cualquier cosa realmente vivida. Por ello somos más ricos o más pobres, tenemos una necesidad más o menos, y en pleno día, incluso en los más serenos instantes de nuestro espíritu despierto, somos llevados un poco de la mano por los hábitos de nuestro sueño (Sánchez Mejías 1988: 59)

Cette dramaturgie du jeu de rêves passe notamment par une «stratégie de l'écart» entre l'austérité de l'espace dramatique du premier acte et la plus que suspecte "esplendor del decorado» qui «ha de llamar la atención» dans l'acte qui suit. La didascalie dit alors l'entrée dans l'illusion onirique. Les indications scéniques rappellent sans cesse la condition usurpée de chaque personnage, la fausse identité que l'image scénique ne permet plus de déceler: "un loco fantásticamente vestido de soldado», "un loco vestido de criado».

En accord avec un espace luxueux à outrance qu'inaugure le deuxième acte, l'artificialité qui dénote l'univers onirique se lit à travers l'excès qui marque l'image du nouveau fou. La reine "viste lujusamente» et arbore un "porte de verdadera realeza» (Sánchez Mejías 1988: 75). La musique qui accompagne son entrée en scène martèle la vision merveilleuse tout droit inspirée de l'univers du conte et qui était annoncée dès la fin du premier acte: «Vamos a crear una leyenda y darle vida... ». (Sánchez Mejías 1988: 63)

La structure psychodramatique semble donc s'effacer lorsque se lève le rideau qui ouvre le deuxième acte. Ainsi, le médecin qui installe avec Don Manuel la situation de théâtre dans le théâtre au premier acte apparaît comme un fou parmi les fous au deuxième acte, la blouse blanche servant alors de déguisement au même titre que la soutane de l'évêque. La machinerie qui lierait l'action à un procédé curatif laisse place à une illusion qui tient plus du rêve éveillé que de la mise en scène puisque tous les changements d'espaces, de costume et donc de conditions sont rejetés dans le hors-scène. N'est alors visible que le fantasme de la vie rêvée des déments. 


\section{L'espace psychiatrique}

L'espace clos de l'hôpital psychiatrique constitue l'élément dramaturgique le plus apparent de cette empreinte freudienne que l'on retrouve dans beaucoup de pièces de la veine du «théâtre psychanalytique» des années 1920 et 1930. Face à la matérialité de la scène, la folie psychique, par essence peu visible, est suggérée à travers le symbole. Ainsi, la didascalie qui ouvre le premier acte annonce l'ambiance angoissante qui règne dans l'asile:

Al levantarse el telón, sala y escenario están completamente a oscuras. Siluetados, con pasta luminosa, se ven todos los aparatos de un laboratorio moderno. Entre ellos se mueven las tres batas blancas de dos médicos y un ayudante.

On est alors plus dans l'évocation d'une ambiance que dans la construction d'un univers mimétique. La déréalisation symboliste passe par le refus du localisme qui universalise la représentation. Le laboratoire moderne que l'on entrevoit grâce à la pâte phosphorescente qui dessine les lignes du mobilier médical et grâce aux blouses blanches impersonnelles suggère une réalité angoissante qui ne disparaît pas même dans l'obscurité. Le clair-obscur qui en résulte évoque une réalité psychiatrique qui ne laisse aucun répit au dément.

Les jeux de lumières sont particulièrement intéressants dans cette dramaturgie symboliste. La lumière crée des jeux scéniques qui glosent l'action et écartent de la sorte cette dramaturgie d'un théâtre réaliste. L'obscurité dans laquelle est plongée la scène au début de l'œuvre dit l'univers psychiatrique angoissant mais aussi l'endroit où l'on cache ce que l'on ne saurait montrer. La folie est dès le départ pointée du doigt comme exclusion, incomprise elle est rejetée dans l'ombre. Cette obscurité laisse place à la lumière du jour qui éclaire une réalité qui peut être montrée, celle des médecins et de l'univers médical.

\section{Mise en scène de la cure psychanalytique}

À l'écriture dramatique de la cure répond une réalité dramaturgique qu'il convient ici de souligner. La nouvelle fonction du docteur implique une gestualité que le dramaturge ne cesse de rappeler. Elle apparaît tout d'abord lors de la première séance avec l'évêque. La didascalie suggère alors la dimension visuelle de l'acte médical: «Le pone la mano izquierda sobre la frente y con la otra se dispone a tomar notas en sus cuartillas, después de poner el reloj sobre la mesa» (Sánchez Mejías 1988: 84). 
La même gestualité alors associée à la cure pour le lecteur-spectateur se répète avec la reine: "La echa en el diván." puis un peu plus loin "le apoya una mano en la frente» (Sánchez Mejías 1988: 90-91). Il y a donc une véritable mise en scène de la cure dans l'œuvre de Sánchez Mejías.

\section{Empreinte élogieuse ou sceptique?}

\section{Du psychanalyste à l'apprenti sorcier}

L'empreinte freudienne dans Sinrazón s'incarne dans une représentation équivoque du médecin psychanalyste, dont la représentation n'est pas anodine. À travers le regard déshumanisé du médecin, le fou est un cas clinique avant d'être un patient dans les mots du docteur Ballina. Les fous sont réduits à de simples objets d'étude et le processus de la cure psychanalytique est unilatéral "hice después el psicoanálisis». Le rapport sujet/objet que constitue la relation médecin/patient dans l'œuvre est confirmé par les nombreux verbes d'actions dont le docteur est le seul sujet «hice», «quedé», «fui», «creí», «le descubrí».

L'image du médecin est alors celle parodique de l'apprenti sorcier, qui joue avec ses créatures, et génère un rire provoqué par le fameux concept de "mécanique plaquée sur du vivant» bergsonien (Bergson 1997: 26). La rigidité qui est ici risible est celle du médecin qui applique une nouvelle théorie du psychisme humain sans égard pour le patient transformé en véritable "machine pathologique»: "Bastó... para que al momento se iniciara el proceso de su curación... Luego, poco a poco, fui sacándole del cuerpo... cuando creí llegado el momento oportuno, le descubrí con violenta sinceridad». (Sánchez Mejías 1988: 59-60)

L'aspect mécanique de la cure psychanalytique est renforcé par la dimension corporelle accordée à la maladie psychique. Le médecin traite l'esprit comme un corps, il en fait son objet. Les actions se succèdent dans un domaine où l'on attend justement la parole et non l'action. Le personnage de Ballina correspond dès lors assez mal à la figure de l'analyste puisqu'il s'agit pour lui de "convaincre» son patient d'une vérité qui n'émane pas d'une prise de conscience du malade mais d'interprétations libres du médecin.

Cette représentation du psychanalyste laisse entrevoir un rejet du dogme psychanalytique dont le médecin fera les frais à la fin de la pièce. 


\section{Le rejet du dogmatisme psychanalytique}

La parodie de l'apprenti sorcier psychanalyste inspire une confiance limitée dans la thérapeutique freudienne. Le théâtre prend d'ailleurs ses distances vis-à-vis des nouvelles théories sur le psychisme humain. En effet, ces passages cités presque textuellement des écrits freudiens sèment le doute quant à la position du dramaturge sur la question. La théorie n'est rapportée dans les propos du docteur que de manière vague et confuse: «[...] le hablé del inconsciente, de la censura que forma la moral, de las luchas entre la conciencia y la inconsciencia, de que su propia enfermedad demostraba su hombría, etcétera, etcétera» (Sánchez Mejías 1988: 60)

L'instrumentalisation de la psychanalyse conjuguée avec la mise en scène de la cure qui peut paraître grotesque pointe du doigt une critique acerbe de la nouvelle thérapeutique.

L'idée d'une vulgarisation de la pensée freudienne suggérée dans une première lecture laisse place à celle d'une vision beaucoup plus critique à l'égard de telles théories. Sánchez Mejías était sans nul doute un fervent admirateur de Freud comme il l'a souligné à maintes reprises: «Hice una comedia freudiana, porque yo leo y admiro al creador del psicoanálisis desde hace años» (Amoros/ Fernández Torres 2010: 297). Cependant, la fin tragique du docteur clame l'échec patent de la psychanalyse comme thérapeutique de la folie. Le dogmatisme de la raison est fortement critiqué parce que «la locura es más fuerte que la razón » (Amoros / Fernández Torres 2010 : 297). Cette raison scientifique qui est mise en échec par une folie qu'on ne parvient pas à soigner ouvre la porte à un discours antipsychiatrique très critique.

L'image qui en résulte est celle d'une folie considérée comme un moindre mal et que la médecine rend tragique par l'enfermement et la stigmatisation. Cette folie vue comme une nécessité vitale à l'homme est montrée par l'état misérable dans lequel se trouve l'évêque au début du troisième acte quand le médecin l'a retiré de force du monde de ses illusions:

En escena, el DOCTOR BALLINA con bata de trabajo, y el oвispo, que viste traje negro de paisano en medio uso y botas del mismo color. Todo en él es desorden. Barba de varios días, camisa sin cuellos ni puños. Cabeza rapada y aspecto triste y resignado. EL DOCTOR observa al OBISPO, que pasea por la sala.

BALLINA- La alegría que producirá a tu padre verte ya en vías de curación. Porque tú te curas. ¡Ya lo creo que te curas! Tú eres hombre de gran 
voluntad, y observo claramente que la has puesto toda al servicio de tu salud. Estoy muy contento contigo. Muy contento.

Le désordre qui prédomine dans cette image scénique rappelle la première apparition de la reine dans l'œuvre figurant ainsi un retour à l'état de manque et d'incomplétude qui est associé à une existence trop raisonnable. Le fou dépourvu de son costume et de l'outrance folklorique n'est que tristesse et résignation. Cette folie nécessaire se fait d'autant plus ressentir que le médecin adopte une attitude en totale inadéquation avec le ressenti de son patient démontrant ainsi l'incommunication qui s'instaure entre les deux. Cette scène incarne le paradoxe que l'on retrouve dans beaucoup de pièces contemporaines où : "Il y a une folie rationnelle ou raisonnable et une rationalité folle ou insensée» (Smadja 2004:267).

Ce scepticisme à l'égard de la psychanalyse serait bien sûr à remettre en contexte, il est loin s'en faut ou pas seulement une prise de position personnelle quant aux idées freudiennes. Bien au contraire il reflète, en tant, que traduction dramatique, la prise de distance qui est celle des intellectuels et psychiatres espagnols de l'époque vis-à-vis des nouvelles théories sur le psychisme humain.

\section{Conclusion}

$\mathrm{Au}$ terme de cette analyse il apparaît que l'empreinte freudienne dans Sinrazón est multiple et qu'elle se répercute à tous les niveaux de l'écriture théâtrale. Cette empreinte constitue une sorte d'hommage distancié ou sceptique rendu par le dramaturge à l'un des pères fondateurs de la psychanalyse.

S'interrogeant sur les moyens de guérison de la folie psychotique, la pièce Sinrazón ne condamne pas la psychanalyse en tant que théorie mais en tant que thérapeutique. L'œuvre place la psychanalyse sur le même plan que l'analyse divine ou biologique de la folie comme une grille d'analyse désuète dont les dérives sont condamnables.

Ce faisant, elle démontre que la normalité n'est qu'une question de point de vue et fustige l'intransigeance de ceux qui souhaitent imposer le leur. C'est à ce titre que le personnage de Don Manuel partage la condition de héros tragique avec le docteur Ballina.

Le combat contre la folie que mènent les deux protagonistes du drame ne peut que finir tragiquement dans la mesure où règne l'incompréhension face à une folie qui est plus humaine que pathologique. L'image qui en ressort 
est celle d'une folie dont on ne peut guérir, qui ne possède pas de thérapie parce qu'elle fait partie inhérente de l'être. Subsiste alors l'idée d'un vécu traumatique avec lequel l'être doit composer. Plutôt que vivre dans l'illusion irréaliste qui comporte des dangers, ce théâtre ne pointe-il pas du doigt la nécessité d'adapter ses traumatismes à son environnement? L'acceptation de la maladie mentale sur scène ne constitue-elle pas le premier pas d'une intégration de ceux que l'on a toujours tenus pour marginaux?

\section{Bibliographie}

Amoros A., Fernández Torres A., 2010, Ignacio Sánchez Mejías, el hombre de la edad de plata, Madrid, Almuzara, 398 p.

Bergson H., 1997, Le Rire: Essai sur la signification du comique, Paris, PUF, 158 p.

Blanco Dávila C., 2000, "Sin Razón y Locura en el Teatro español de Vanguardia», in: Literatura y Sociedad: el papel de la literatura en el siglo $\mathrm{XX}$, [actas del primer congreso nacional "Literatura y Sociedad", A Coruña, Fidel López Criado, p. 341-360.

Freud S., 1985, Le rêve et son interprétation, Paris, Gallimard, 119 p.

Freud S., 2001, Cinq leçons sur la psychanalyse, Paris, Payot \& Rivages, 187 p.

Sánchez Mejías I., 1988, Teatro, Gallego Morell, Antonio, Madrid, Espasa-Calpe, col. «Austral», 184 p.

Sánchez Mejías I., 1928, Sinrazón: juguete trágico en tres actos, Madrid, Prensa Moderna, col. «El Teatro Moderno», año IV, núm. 145, 47 p.

Sánchez Mejías I., 2010, Sinrazón: juguete trágico en tres actos, Sevilla, Espuela de Plata, col. «El Teatro Moderno», 85 p.

Sarrazac J.P., 2004, Jeux de rêve et autres détours, Paris, Circé, 141 p.

Smadja I., 2004, La folie au théâtre: regards de dramaturges sur une mutation, Paris, PUF, 347 p.

Thuillier J., 1996, La Folie, Histoire et dictionnaire, Paris: Robert Laffont, 827 p. 